\title{
Arrestin in Ciliary Invertebrate Photoreceptors: Molecular Identification and Functional Analysis In Vivo
}

\author{
Maria del Pilar Gomez, ${ }^{1,5}$ Lady Espinosa, ${ }^{2}$ Nelson Ramirez, ${ }^{3}$ and Enrico Nasi ${ }^{4,5}$ \\ ${ }^{1}$ Department of Biology, ${ }^{2}$ Graduate Program in Biotechnology, ${ }^{3}$ Graduate Program in Neuroscience, and ${ }^{4}$ Institute of Genetics, Universidad Nacional de \\ Colombia, Bogotá 111321, Colombia, and ${ }^{5}$ Marine Biological Laboratory, Woods Hole, Massachusetts 02543
}

Arrestin was identified in ciliary photoreceptors of Pecten irradians, and its role in terminating the light response was established electrophysiologically. Downstream effectors in these unusual visual cells diverge from both microvillar photoreceptors and rods and cones; the finding that key regulatory mechanisms of the early steps of visual excitation are conserved across such distant lineages of photoreceptors underscores that a common blueprint for phototransduction exists across metazoa. Arrestin was detected by Western blot analysis of retinal lysates, and localized in ciliary photoreceptors by immunostaining of whole-eye cryosections and dissociated cells. Two arrestin isoforms were molecularly identified by PCR; these present the canonical N-and C-arrestin domains, and are identical at the nucleotide level over much of their sequence. A high degree of homology to various $\beta$-arrestins (up to $70 \%$ amino acid identity) was found. In situ hybridization localized the two transcripts within the retina, but failed to reveal finer spatial segregation, possibly because of insufficient differences between the riboprobes. Intracellular dialysis of anti arrestin antibodies into voltage-clamped ciliary photoreceptors produced a gradual slow-down of the photocurrent falling phase, leaving a tail that decayed over many seconds after light termination. The antibodies also caused spectrally neutral flashes to elicit prolonged aftercurrents in the absence of large metarhodopsin accumulation; such aftercurrents could be quenched by chromatic illumination that photoconverts metarhodopsin back to rhodopsin. These observations indicate that the antibodies depleted functionally available arrestin, and implicate this molecule in the deactivation of the photoresponse at the rhodopsin level.

\section{Introduction}

In any sensory cell, the ability to respond faithfully to changing stimulation requires mechanisms for quenching the excitatory cascade when stimulation subsides. In a multistage signaling pathway, each individual step must have its own shutoff mechanism, beginning with the receptor molecule; this is the case of different types of visual cells (reviewed by Yau and Hardie, 2009; Fain et al., 2010), where the photopigment belongs to the superfamily of hepta-helical receptors. In vertebrate rods and cones the inactivation of photoisomerized rhodopsin is initiated by phosphorylation, followed by binding to arrestin, which prevents further interaction with G-proteins (for review, see Palczewski, 1994). Arrestin orthologs have also been identified in microvillar photoreceptors of several invertebrates, such as Drosophila (Hyde et al., 1990; LeVine et al., 1990; Smith et al., 1990; Yamada et al., 1990), Calliphora (Bentrop et al., 1993; Plangger et al., 1994), Limulus (Smith et al., 1995), and Loligo (Mayeenuddin and Mitchel, 2003). This class of invertebrate visual cells thus contains elements similar to the molecular machinery used by vertebrates for photopigment deactivation.

\footnotetext{
Received June 28, 2010; revised Nov. 23, 2010; accepted Dec. 2, 2010. This work was supported by National Science Foundation Grant 0639774.

Correspondence should be addressed to Dr. Enrico Nasi, Marine Biological Laboratory, 7 MBL Street, Woods Hole, MA 02543. E-mail: enasi@mbl.edu.

DOI:10.1523/JNEUROSCI.3320-10.2011

Copyright $\odot 2011$ the authors $\quad 0270-6474 / 11 / 311811-09 \$ 15.00 / 0$
}

The retina of certain mollusks possesses not only the canonical microvillar photoreceptors found in other invertebrates, but also an additional, distinct class that constitute a third lineage of lightsensing cells among metazoa. These visual receptors resemble rods and cones in terms of (1) the ciliary origin of the lightsensing structure (Miller, 1958; Barber et al., 1967), (2) the hyperpolarizing receptor potential (Gorman and McReynolds, 1969), and (3) the role of cGMP as an internal messenger controlling the light-sensitive conductance (Gomez and Nasi, 1995); nonetheless, their transduction cascade diverges in several key respects: in the first place, light stimulation brings about membrane hyperpolarization by opening, rather than closing, ion channels that are gated by cGMP, like those of rods and cones, but are selective for potassium ions (Gorman and McReynolds, 1978; Gomez and Nasi, 1994). This calls for a lighttriggered elevation of cGMP, instead of a decrease. Second, the light-regulated enzyme responsible for changing cGMP levels appears to be a cyclase, instead of a phosphodiesterase (Gomez and Nasi, 2000). Finally, the rhodopsin-regulated G-protein is not transducin, but belongs to the $G_{o}$ subtype (Kojima et al., 1997). As such, these cells deviate from other known light receptors. The mechanisms involved in the termination of the photoresponse in ciliary invertebrate photoreceptors had not been previously investigated. In the present report we detected arrestin in Pecten retinae, determined its localization in ciliary photoreceptors, molecularly identified two isoforms of arrestin, and garnered functional evidence for its role in quenching the light-activated current. The results are discussed in the light of the evolutionary history of visual 
mechanisms, and the phenomenology of sustained visual excitation in response to chromatic photostimulation.

\section{Materials and Methods \\ Electrophysiology}

Specimens of hermaphrodite bivalve mollusk Pecten irradians were obtained from the Aquatic Resources Division of the Marine Biological Laboratory (Woods Hole, MA). The techniques for enzymatically isolating viable ciliary photoreceptors and performing whole-cell patch-clamp recording have been described in detail (Gomez and Nasi, 1994). Cells plated in a flow-chamber were continuously superfused with artificial sea water (ASW) containing (in mM): $480 \mathrm{NaCl}, 10 \mathrm{KCl}, 10 \mathrm{CaCl}_{2}, 49 \mathrm{MgCl}_{2}$, 10 HEPES, $5.5 \mathrm{D}$-glucose, $\mathrm{pH}$ 7.75. The "intracellular" solution used to fill thin-wall borosilicate patch pipettes (Garner Glass) contained (in mM): $100 \mathrm{KCl}, 200 \mathrm{~K}$-glutamate, $22 \mathrm{NaCl}, 5 \mathrm{Mg}$ ATP, 10 HEPES, 1 EGTA, 100 $\mu \mathrm{M}$ GTP, and 300 sucrose, $\mathrm{pH}$ 7.3. Electrode resistance, measured in ASW, was $2-4 M \Omega$; series resistance was routinely compensated. Current signals were low-pass filtered at $1 \mathrm{kHz}(-3 \mathrm{~dB})$ with a Bessel 4-pole filter, before digitizing at $3 \mathrm{kHz}$ sampling rate with 12-bit resolution (Data Translation DT-3000). Software developed in-house was used for data acquisition, stimulation, and off-line analysis. Intracellular application of anti-arrestin antibodies (Abs) was accomplished by dialysis through the patch pipette; the tip of the microelectrode was prefilled by dipping with antibody-free solution to prevent interference with seal formation.

\section{Light stimulation}

The standard optical stimulator consisted of a $100 \mathrm{~W}$ tungsten-halogen light source (Oriel), with the output beam combined with that of the microscope illuminator via a beam splitter prism placed above the condenser. Alternatively, for stronger chromatic photostimulation, a $100 \mathrm{~W}$ $\mathrm{Hg}$ arc lamp (Zeiss) was coupled to the epifluorescence port of the microscope via a liquid light-guide (Oriel). In both cases, a condenser, an infrared absorbing filter, an electromechanical shutter (Vincent Associates), and collimating and field lenses and filters were interposed in the light path, while an adjustable pinhole or an iris diaphragm placed in a conjugate image plane restricted the illuminated region on the recording chamber to a disc $\sim 200 \mu \mathrm{m}$ in diameter. Unless otherwise specified, broad-band light was used (515-670 $\mathrm{nm}$ ), determined by the combination of a heat-absorbing filter and an edge filter (Schott Glass Technologies) interposed in the light path. Effective light intensity was calibrated in vivo by matching the photocurrent amplitude to that obtained with monochromatic light (500 nm, 3-cavity interference filter; Ditric Optics), the intensity of which was measured with a radiometer (UDT, model 370). Light intensity is expressed in terms of equivalent photon flux at $500 \mathrm{~nm}$ (Gomez and Nasi, 1994; Gomez and Nasi, 1995). Calibrated neutral-density filters (Melles Griot) provided controlled light attenuation. During experimental manipulations the cells were viewed with an infrared-sensitive Newvicon TV camera (model WV-1550; Panasonic) using a near-IR long-pass filter for illumination $(\lambda>780 \mathrm{~nm}$; Andover Corporation). The infrared illuminator was turned off for several minutes before testing light responses.

\section{Immunodetection}

Antibodies. Several polyclonal antibodies targeting visual arrestin or $\beta$-arrestin of vertebrate origin were tested. All were affinity purified. Three of them (E-12, N-19, and H-290, raised against human arrestins) were obtained from Santa Cruz Biotechnology; a fourth antibody against amphibian visual arrestin was kindly provided by Dr. Harris Ripps, University of Illinois (Chicago, IL) (a48K, which has wide species crossreactivity and also cross-reacts with $\beta$-arrestin; Dr. H. Ripps, personal communication). When used for intracellular dialysis, the final concentrations of $\mathrm{Na}$ azide was $0.0005 \%$.

Western blots. Retinas ( $\approx 20 /$ lane) were homogenized (Teflon/glass) in the presence of protease inhibitors (100 $\mu \mathrm{M}$ PMSF and 0.1\% Sigma Protease Inhibitor Cocktail), acetone-precipitated for $2 \mathrm{~h}$ at $-20^{\circ} \mathrm{C}$, and centrifuged 10 min at $10,000 \times g$. The pellet was air-dried, resuspended in sample buffer, and separated by SDS-PAGE (12\%). Proteins were electrotransferred ( $1 \mathrm{~h}, 100 \mathrm{~V}$, Bio-Rad Mini Trans-blot) onto a nitrocellulose membrane which was then blocked overnight with $3 \%$ BSA. The membrane was then sequentially incubated with primary antibodies (1: 2000 to $1: 500,1-3 \mathrm{~h}$ ), washed three times in Tris-buffered saline and incubated in alkaline phosphatase-conjugated secondary Abs (Promega; 1:2000, $1 \mathrm{~h}$ ). After the final washes, the nitrocellulose membrane was developed in Western Blue (Promega).

Immunohistochemistry. Whole eyes were excised and fixed overnight in $90 \% \mathrm{ASW}$ containing $4 \%$ paraformaldehyde at $4^{\circ} \mathrm{C}$. Following permeabilization (PBS $+0.2 \%$ Triton-X, 5 min on ice) and 3 steps of sucrose impregnation ( $10 \% \rightarrow 20 \%$ for $3-4 \mathrm{~h}$ each, then $30 \%$ overnight), eyes were embedded in gelatin $\left(7.5 \%+15 \%\right.$ sucrose), chilled to $-20^{\circ} \mathrm{C}$, and sectioned at $12 \mu \mathrm{M}$ in a cryostat (Thermo Scientific Microm HM 505-N). Slices were plated onto electrostatically charged glass slides (Fisher Scientific Superfrost Plus), dried, and kept at $-20^{\circ} \mathrm{C}$ for subsequent use. After gelatin removal by immersion for $5 \mathrm{~min}$ in PBS warmed to $36^{\circ} \mathrm{C}$, cryosections were blocked for 30 min with $1 \%$ goat or horse serum (depending on the antibodies to be used) diluted in PBS and supplemented with $0.02 \%$ Triton-X. Incubation with primary antibodies (dilution $1: 1000$ to $1: 200$ ) in PBS $+0.5 \%$ BSA (40 min to $2 \mathrm{~h}$ ) was followed by 2 washes in PBS. For detection, the ABC Elite kit from Vector Labs was used, following the manufacturer's indications. Briefly, the samples were treated with biotinylated secondary Abs for $30 \mathrm{~min}$ and then incubated with streptavidin-conjugated peroxidase for $30 \mathrm{~min}$. After washing and development in Vector VIP substrate and washing with distilled water, the slides were dehydrated with ethanol $(\mathrm{EtOH})$ in several steps $(30 \%$, $50 \%, 70 \%, 95 \%$, and $2 \times 100 \%$ ) and incubated twice in Xylenes ( $5 \mathrm{~min}$ each) before mounting with Permount. For visualization, a Zeiss Axioplan microscope equipped with Nomarski optics and a digital camera (Zeiss Axiocam) were used.

Immunocytochemistry. Enzymatically dispersed retinal cell suspensions were plated onto coverslips pretreated with concanavalin A (5 $\mathrm{mg} / \mathrm{ml}$ in $1 \mathrm{M} \mathrm{NaCl}, 3 \mathrm{~h}$ ). Cells were fixed in ASW containing $2 \%$ paraformaldehyde for $15 \mathrm{~min}$, washed three times in $0.1 \mathrm{M}$ PBS, and permeabilized in $0.2 \%$ Triton-X for $5 \mathrm{~min}$ on ice. Antibody treatment followed steps similar to those described above, except that in some assays, fluorescent antibodies (conjugated to Alexa Fluor 488 or Alexa Fluor 546; Invitrogen) were used at a dilution of 1:200, and, after the final washes (3X in PBS), slides were mounted with coverslips using 50\% glycerol/ PBS, and sealed with nail polish. For fluorescence images, either a fluorescein (BP 470/40, FT 495 dichroic reflector, BP $525 \mathrm{~nm}$, Zeiss) or a rhodamine (BP $546 \mathrm{~nm}$, FT $580 \mathrm{~nm}$, and a LP $590 \mathrm{~nm}$ long-pass emission filter) filter set was used. Exposure was first adjusted with control slides until autofluorescence virtually disappeared; those same settings were then used for the corresponding experimental slides.

PCR cloning. Pecten retina cDNA was obtained from poly(A) mRNA isolated by magnetic microbeads covalently attached to oligo(dT) (Dynal). For reverse transcription we used the SMART RACE (rapid amplification of cDNA ends) kit (Clontech), which yields 5' and 3' RACEready cDNAs with anchor sequences added at either end. Degenerate primers, based on conserved domains from multiple amino acid alignments, were designed either manually or with the aid of the programs Block Maker and CodeHop. PCR amplifications were performed on an MJ Research DNA Engine cycler, using Taq polymerase (New England Biolabs). Amplicons were checked on agarose gels and purified as needed (Qiagen Qiaquick PCR or Gel Purification kits). For product extension, we used nested RACE reactions, combining gene-specific nondegenerate primers (designed with Primer3 on the basis of obtained nucleotide sequences) and "universal" anchor primers. Upon obtaining overlapping sequences that jointly span full-length clones (as assembled with the CAP (Contig Assembly Program) routine in the BioEdit software package), the whole product was amplified in a single end-to-end run, to rule out spurious amplification of noncontiguous cDNAs. For sequencing, purified PCR products were ligated into pGEM-T Easy TA vector (Promega). Chemically transformed Escherichia coli were grown overnight in ampicillin agar plates with isopropyl- $\beta$-D-thiogalactopyranoside and X-gal; individual colonies were picked and cultured overnight, and rechecked by bacterial colony PCR. Plasmid DNA was recovered using Qiagen spin columns, subjected to restriction analysis, and sent out for sequencing (Cornell Bio-Resource Center, Ithaca, NY). 


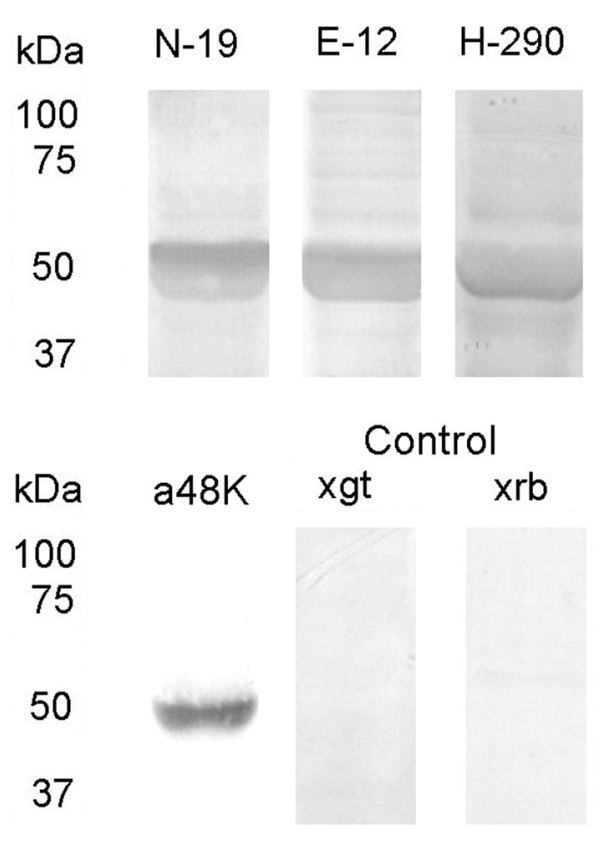

Figure 1. Western blot of scallop retinal tissue. Retinal proteins separated on SDS-PAGE (12\%) were electrotransferred to nitrocellulose membranes and incubated (1:1000) with several antibodies raised against vertebrate arrestins, followed by secondary Abs conjugated to alkaline phosphatase, and development. All antibodies produced a band of similar apparent molecular mass, $\approx 50 \mathrm{kDa}$. Omission of the primary antibodies and incubation with either anti-goat $(\times \mathrm{gt})$ or anti-rabbit $(\times \mathrm{rb})$ alkaline phosphatase-conjugated antibodies did not produce any signal.

\section{In situ hybridization}

Probe generation. Target sequences containing suitable unique restriction sites were amplified by PCR, digested with restriction enzymes, and directionally ligated into a dual-promoter expression vector (Stratagene Bluescript II). Plasmids were linearized and incubated in digoxigenin labeling mix with RNA polymerase (T7 or T3, to generate either sense or antisense probe). The reaction was stopped with EDTA, $\mathrm{pH}$ 8.0. After checking the RNA in an agarose gel, the probe was diluted in $600 \mu \mathrm{l}$ of EtOH, $25 \mu \mathrm{l}$ of $3 \mathrm{M}$ $\mathrm{NaAc}$, and $240 \mu \mathrm{l}$ of DEPC $\mathrm{H}_{2} \mathrm{O}$, and incubated 5 min on ice. EtOH was removed by spinning at $14,000 \times g$ for $30 \mathrm{~min}$ at $4^{\circ} \mathrm{C}$, drying in a SpeedVac, and resuspending in $20 \mu \mathrm{l}$ of DEPC $\mathrm{H}_{2} \mathrm{O}$. After measuring an aliquot in a spectrophotometer, the sample was diluted in hybridization buffer to $10 \times$ stock concentration $(20 \mu \mathrm{g} / \mathrm{ml})$ and stored at $-20^{\circ} \mathrm{C}$.

Probe detection. Eye cryosections were sequentially washed in PBS, methanol, and PBS + Tween $20(\mathrm{PTw})$. After treatment with proteinase $\mathrm{K}$ in PTw and washing with glycine in PTw $(2 \mathrm{mg} / \mathrm{ml})$, the cryosections were refixed in $0.2 \%$ glutaraldehyde $+4 \%$ paraformaldehyde in PBS. Following washing, they were treated with $0.1 \%$ sodium borohydride in PTw, washed again in PTw and in hybridization buffer, and prehybridized for $45 \mathrm{~min}$ at $60^{\circ} \mathrm{C}$. Subsequently, the probe was added, and the slides were coverslipped (HibriSlips, Grace Bio) and incubated overnight at $60^{\circ} \mathrm{C}$ in a hybridization oven (Boekel Scientific). After prying off the coverslip, washing repeatedly, and rinsing in NTE $(0.5 \mathrm{M} \mathrm{NaCl}, 10 \mathrm{~mm}$ Tris pH 8.0, 1 mm EDTA), the slides were incubated with RNase A and RNase T1 in NTE, washed again, and transferred to a humidified dark container. Sections were blocked off with a PAP pen, and rocked in the presence of levamisole and Boehringer Mannheim Blocking Reagent. The solution was replaced with anti-digoxigenin AP antibody (1:2000) and incubated overnight. For probe detection, the slides were washed and AP substrate solution (Roche) was added, followed by a final wash.

\section{Results}

Immunodetection of arrestin in Pecten visual cells

To establish whether proteins akin to vertebrate arrestin isoforms exist in Pecten eyes, Western blot analysis was performed on retina lysates. Figure 1 shows the outcome obtained with four

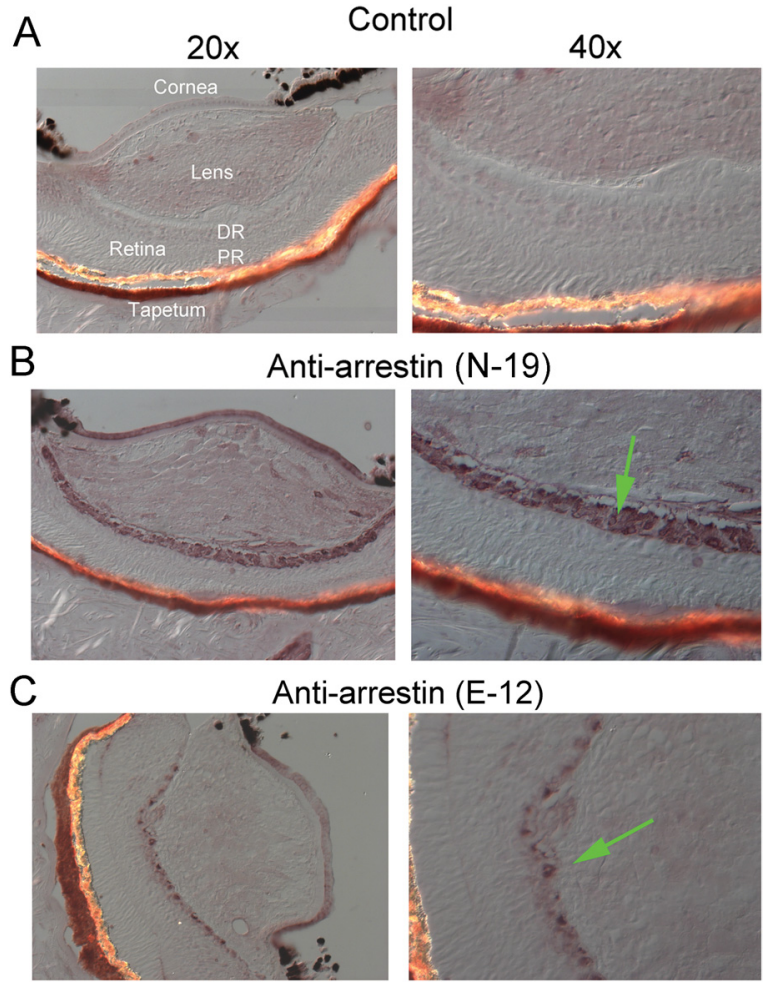

Figure 2. Immunolocalization of arrestin in Pecten eye. $A$, Nomarski micrographs of a control $12-\mu \mathrm{m}$-thick cryosection through a whole scallop eye, fixed in paraformaldehyde. The section was incubated only with secondary antibodies, and is displayed at two magnifications (RD, distal retina; PR, proximal retina). $\boldsymbol{B}$, Section treated with one of the anti-arrestin antibodies (N-19); a distinct immunostaining pattern is observed, which is confined to the distal retinal layer, where the ciliary, hyperpolarizing photoreceptors are located (arrow in lower right panel). C, Staining by the E-12 antibody was also segregated to the distal retina.

different anti-arrestin polyclonal antibodies raised against vertebrate species. In all cases, a prominent band of the expected molecular weight $(\approx 50 \mathrm{kDa})$ was obtained, with little contamination by background staining or bands of different apparent mass. Control lanes, only treated with the two secondary AP-conjugated antibodies produced no detectable signals. We then turned to immunohistochemistry in fixed sections, to determine the tissue localization of the anti-arrestin immunoreactivity within the eye. All the antibodies stained the retina with varying intensity, including the distal layer where ciliary photoreceptors are located; in the case of two of the antibodies (E-12 and N-19) a distinct spatial pattern was produced, which selectively decorated the distal retina. An example is illustrated in Figure 2; the bottom panels show Nomarski micrographs of $12-\mu \mathrm{m}$-thick cryosections through a whole scallop eye stained with N-19 and E-12; the antibodies labeled the distal layer of the retina. The cornea was also decorated, but considering the ubiquitous presence of different arrestins across cell types (Gurevich and Gurevich, 2006), this result detracts little from the specificity of the staining pattern within the retina. The top panels show a control slide in which primary antibodies were omitted, and is completely devoid of label.

\section{Single-cell labeling with anti-arrestin antibodies}

To refine the localization of arrestin to identified ciliary visual cells, Pecten retinae were enzymatically and mechanically dispersed. Figure $3 A$ illustrates an isolated distal photoreceptor treated with anti-arrestin antibody (a48k), together with a control cell; the detection was with peroxidase. In $\boldsymbol{B}-\boldsymbol{F}$ of the same figure, the other antibodies were tested in dissociated ciliary photoreceptors. In this case, the detection was by means of secondary 
antibodies conjugated to Alexa Fluor 546. Virtually all bona fide ciliary visual cells of the distal retina examined were immunopositive for arrestin; no obvious spatial segregation of the label could be discerned within the cell, indicating that arrestin is present both in the light-sensitive ciliary appendages and in the soma. In some photoreceptors arrestin has been shown to translocate between the soma and the light-sensitive lobe of the cell (Hardie, 2003). A pilot attempt to compare fixation of ciliary cells in dim red light vs normal room light did not reveal any obvious differential pattern of spatial distribution. However, redistribution may be hard to gauge, because there are cilia emanating over much of the soma (not just those projecting from the apical end, which are readily visualized), an arrangement that can confound the localization of the immunostain.

\section{Anti-arrestin antibodies slow down photocurrent shutoff}

To ascertain the functional role of arrestin in the regulation of the light response of ciliary photoreceptors, we tested the antiarrestin antibodies for potential functional effects in live cells. We exploited the durable recordings that can be obtained under whole-cell clamp in these photoreceptors and applied a48K antibodies by intracellular dialysis via the patch electrodes (diluted 1:200 in the standard internal solution supplemented with $0.1 \%$ BSA as a carrier). Immediately after attaining the whole-cell configuration, the cells were stimulated with repetitive $100 \mathrm{~ms}$ flashes of approximately half-saturating intensity. Figure $4 \mathrm{~A}$ shows superimposed traces recorded at 5 min intervals. During the course of dialysis with anti-arrestin antibodies, the falling phase of the photocurrent became progressively slower and a long-lasting residual tail developed $(n=6)$. By contrast, in control cells (Fig. $4 \mathrm{~A}$, top, right) the fall kinetics only marginally slowed down during the first few minutes after attaining whole-cell clamp, and stabilized thereafter; unlike Ab-treated cells, the photocurrent fully shut off in $<2 \mathrm{~s}$ after the test flash, without any hint of persistent activation $(n=5)$. The slight change in the rising phase of the photocurrent visible in Figure $4 \mathrm{~A}$ was not consistently observed, nor did it associate selectively with the antibody treatment. Figure $4 B$ shows a comparison of the initial flash response and a response after $35 \mathrm{~min}$ of dialysis with the anti-arrestin Abs. Notice that these traces are presented on a compressed time scale, to highlight the duration of the slowly deactivating tail. To quantify these effects, the response duration at half-maximal amplitude [full width at halfmaximum (FWHM)] was measured for the initial photocurrent and after 25 min of recording; the bar-graph in Figure $4 C$ shows the average values obtained in experimental and control cells. The difference in the relative increase in response duration was statistically significant $(p<0.01, t$ test $)$. As an additional control, two photoreceptors were dialyzed with antibodies that were denatured by boiling (10 min), and showed no significant increase in response duration $\left(\Delta_{\mathrm{FWHM}}=13 \pm 9 \%\right)$. The dilution 1:200 did not appear to yield saturating effects: two additional cells were tested with 1:50 dilution of the antibodies, and the increase in response duration appeared to be even more pronounced (an additional $13 \% \pm 9.6 \%$ with respect to the effects of the 1:200 treatment).

The dependency of the steady-state effects of anti-arrestin Abs on the intensity of stimulating light was also examined. Intensity series were measured in photoreceptors treated extensively with anti-arrestin antibodies (>30 min) and compared with control cells. Figure $4 D$ shows representative examples: in experimental cells (left), a long-lasting tail appeared in the photocurrent even at light intensities that elicit responses in the linear range, something we had never observed in control conditions (right), where only upon reaching saturation do the fall kinetics of the photocurrent become protracted.

\section{Prolonged aftercurrents reflect arrestin function}

In many invertebrate photoreceptors the absorption spectra of rhodopsin (R) and metarhodopsin (M) differ significantly, and monochromatic illumination can induce a net shift in pigment distribution which is accompanied by prolonged aftercurrents (PAs) (for review, see Hillman et al., 1983). In Pecten ciliary photoreceptors, $\mathrm{R}$ and $\mathrm{M}$ have $\lambda_{\max }$ of $\approx 500 \mathrm{~nm}$ and $\approx 575 \mathrm{~nm}$, respectively, and intense blue lights (B) evoke PAs (Cornwall and Gorman, 1983). By contrast, long-wavelength flashes (R), no matter how strong, cannot elicit PAs; on the contrary, they can 

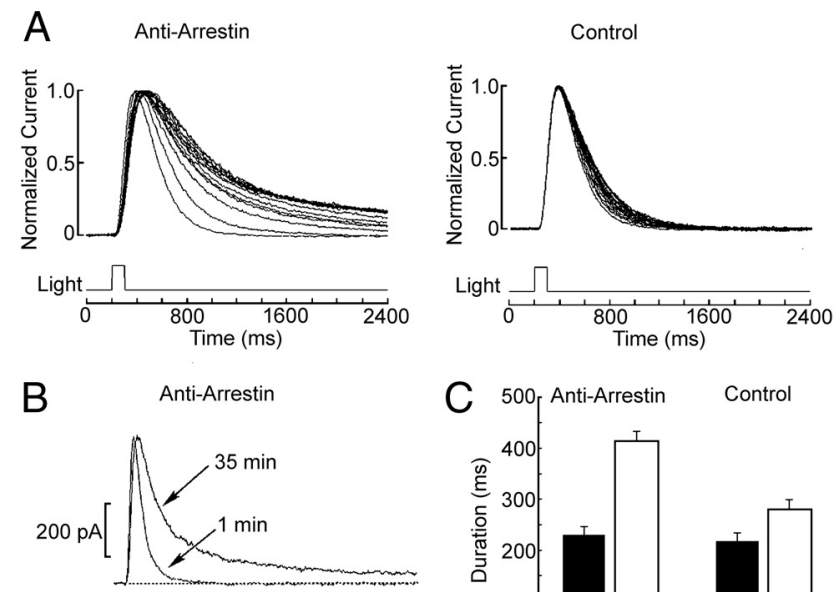

Light $几$
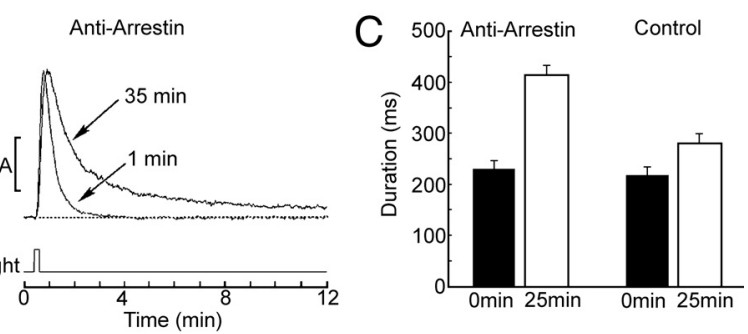

D

Anti-Arrestin
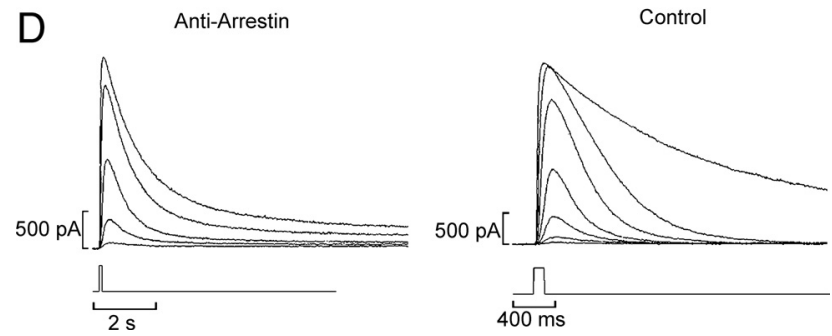

Figure 4. Anti-arrestin antibodies (a48K) slow down photocurrent shutoff. Anti-arrestin antibodies were intracellularly perfused (1:200) into whole-cell clamped ciliary photoreceptors. $\boldsymbol{A}$, Cells were stimulated with repetitive $100 \mathrm{~ms}$ flashes of approximately half-saturating intensity $\left(2 \times 10^{13}\right.$ photons $\left.\cdot \mathrm{cm}^{-2} \cdot \mathrm{s}^{-1}\right)$. Top, Left, Superimposed, normalized photocurrent traces recorded at $5 \mathrm{~min}$ intervals. After $>10 \mathrm{~min}$ of dialysis, the falling phase of the photocurrent became progressively slower. Top, Right, By contrast, in control cells, the fall kinetics only marginally slowed down during the first few minutes of dialysis and stabilized thereafter. $\boldsymbol{B}$, Comparison of the initial flash response and a response after $35 \mathrm{~min}$ of dialysis with the Abs, shown on a compressed time scale, to highlight the duration of the slowly deactivating tail (different cell; light intensity $4.7 \times 10^{13}$ photons $\cdot \mathrm{cm}^{-2} \cdot \mathrm{s}^{-1}$ ). C, Summary of the antiarrestin antibodies effect on the photocurrent kinetics. The duration at half-maximal amplitude (FWHM) of the initial light response and after $25 \mathrm{~min}$ of dialysis was averaged for control cells and for cells dialyzed with anti-arrestin antibodies (1:200); error bars indicate the SE. The difference across the two groups in the relative lengthening of the photocurrent over time was statistically significant at the 0.01 level. D, Slow photocurrent shutoff after antibody treatment, with subsaturating light stimulation. An intensity series with flash intensity incremented at 0.6 log step was measured in a photoreceptor treated extensively with anti-arrestin antibodies (left). Even light responses in the linear range exhibited a long-lasting tail, unlike in control conditions (right), where only upon reaching saturation was the time course of the photocurrent prolonged. Intensity of the unattenuated light: $7.48 \times 10^{14}$ photons $\cdot \mathrm{cm}^{-2} \cdot \mathrm{s}^{-1}$. The attenuation series for the anti-arrestin antibody-treated cell was -3.2 to $-0.8 \log$ at $0.6 \mathrm{log}$ increments, and for the control cell, -3.6 to 0 log at 0.6 log increments. Holding potential, $-30 \mathrm{mV}$ throughout.

reset them by photoconverting $\mathrm{M} \rightarrow \mathrm{R}$. An example is shown in Figure $5 A$ : a ciliary photoreceptor voltage-clamped at a holding potential of $-30 \mathrm{mV}$ was stimulated with a flash of light of $420-$ $480 \mathrm{~nm}$. The photocurrent peaked and decayed to a prolonged tail, several hundred picoamperes in amplitude, which remained stable for over $1 \mathrm{~min}$. When a second flash of $\lambda>640 \mathrm{~nm}$ was applied, the current rapidly returned to baseline. The sustained aftercurrent has been attributed to saturation of the available arrestin, because of the excessive buildup of activated rhodopsin (Dolph et al., 1993).

If anti-arrestin antibodies slow down photocurrent shutoff by functionally impairing arrestin, this manipulation should also
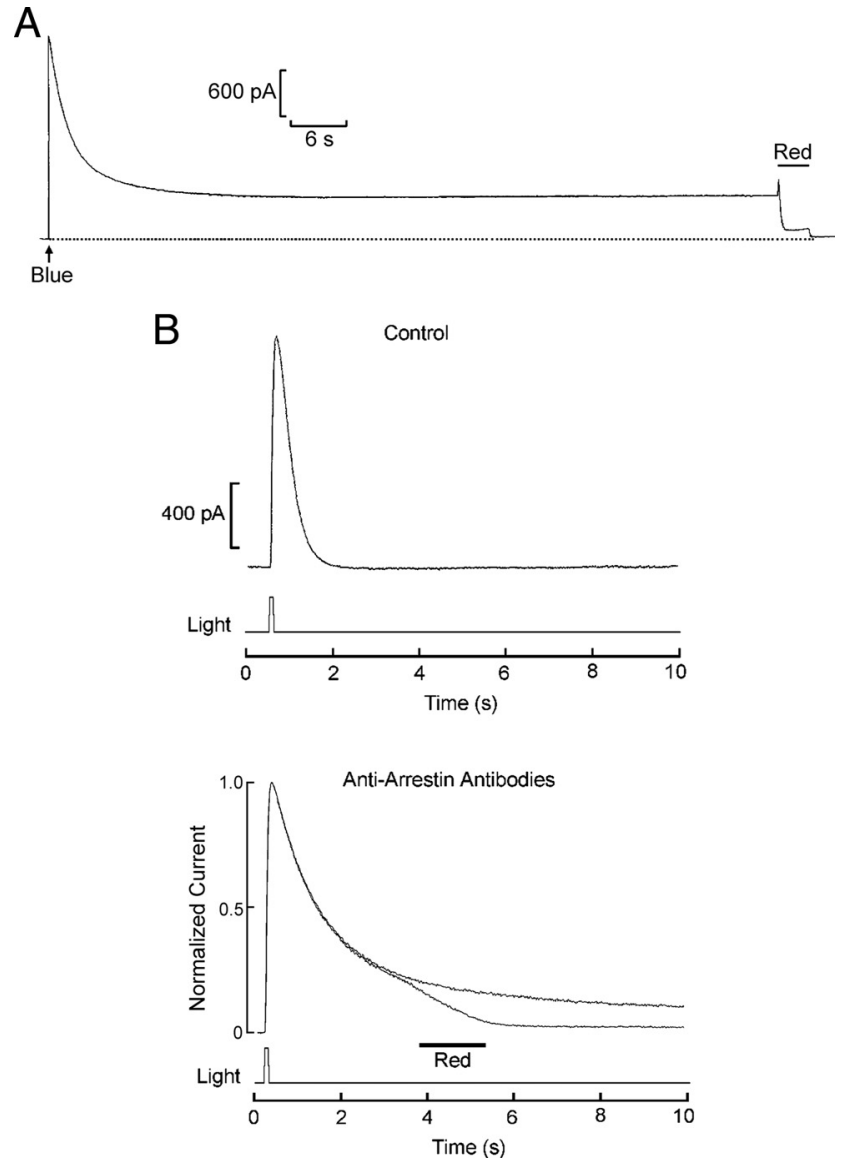

Figure 5. Prolonged aftercurrents reflect saturation of arrestin. $\boldsymbol{A}$, Stimulation with an intense blue flash $\left(1.32 \times 10^{15}\right.$ photons $\left.\cdot \mathrm{cm}^{-2} \cdot \mathrm{s}^{-1}\right)$ evokes a persistent aftercurrent in a ciliary photoreceptor held under voltage clamp at $-30 \mathrm{mV}$. After $\sim 1$ min of sustained excitation, delivery of a long-wavelength light $\left(\mathrm{red} ; 4.7 \times 10^{16}\right.$ photons $\left.\cdot \mathrm{cm}^{-2} \cdot \mathrm{s}^{-1}\right)$ reset the photocurrent by photoconverting $M \rightarrow R$. $B$, Top, Effect of light stimulation of subsaturating intensity in a control cell, using a wavelength near the isosbestic $\mathrm{R} / \mathrm{M}$ wavelength $(\lambda=540$ $\mathrm{nm})$, intended to avoid the generation of a large pool of metarhodopsin. The photocurrent decayed to baseline in $<1.5 \mathrm{~s}$; holding potential $-30 \mathrm{mV}$. Bottom, A similar stimulus was applied to another cell extensively dialyzed with anti-arrestin Abs; in this case, the decay phase of the response was dramatically retarded, but the subsequent delivery of a red light caused the complete shutoff of the lingering photocurrent, indicating that it stemmed from persistent activation of photopigment that could not be quenched by the available arrestin. By contrast, omission of the red light caused the tail to linger for a prolonged period of time.

enhance the cell's propensity to produce prolonged aftercurrents, without requiring a massive accumulation of metarhodopsin. To test this proposition, flashes near the isosbestic $\mathrm{R} / \mathrm{M}$ wavelength were used ( $\lambda=540 \mathrm{~nm}$ ), to minimize the occurrence of a large net shift in pigment state distribution. In Figure $5 B$ (top) we show that such stimuli do not compromise the swift termination of the photoresponse in control cells. By contrast, after perfusion with anti-arrestin Abs (bottom) the photocurrent evoked by $540 \mathrm{~nm}$ flashes was dramatically prolonged, much like the effects of bright blue illumination in control cells $(n=3)$. Administration of red light $(\mathrm{R})$ terminated the lingering current, demonstrating that it was indeed due to persistent rhodopsin activation; in a corresponding trace without the red stimulation, by contrast, the tail continues.

\section{Molecular cloning of two arrestin isoforms from the retina of Pecten}

We also sought to determine the molecular identity of arrestin in the retina of Pecten. Degenerate primers were designed on the 


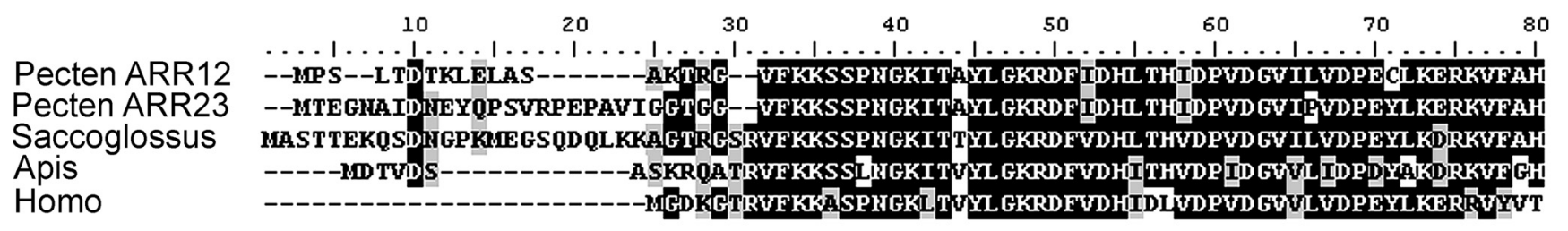

Pecten ARR12

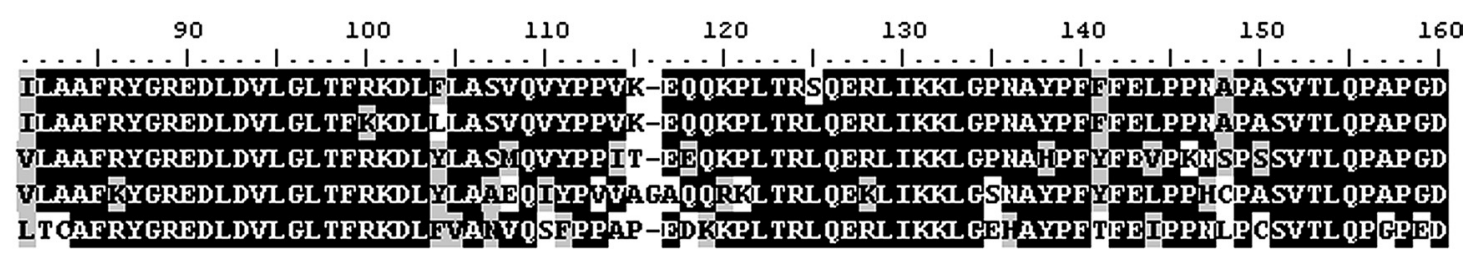

Pecten ARR23

Apis

Homo

Pecten ARR12

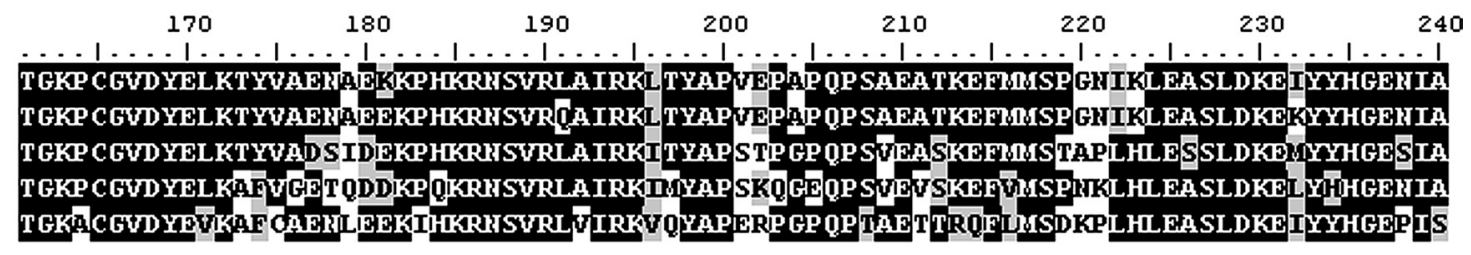

Pecten ARR12

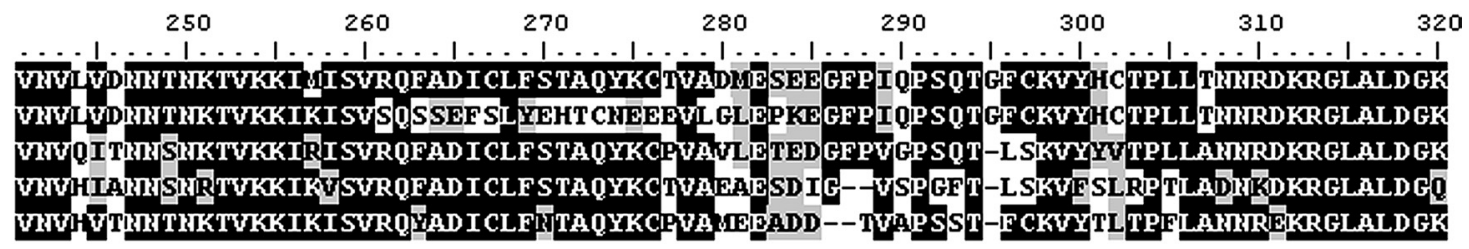

Pecten ARR23

Apis

Homo

Saccoglossus

Apis

Homo

Pecten ARR12
Pecten ARR23
Saccoglossus
Apis
Homo

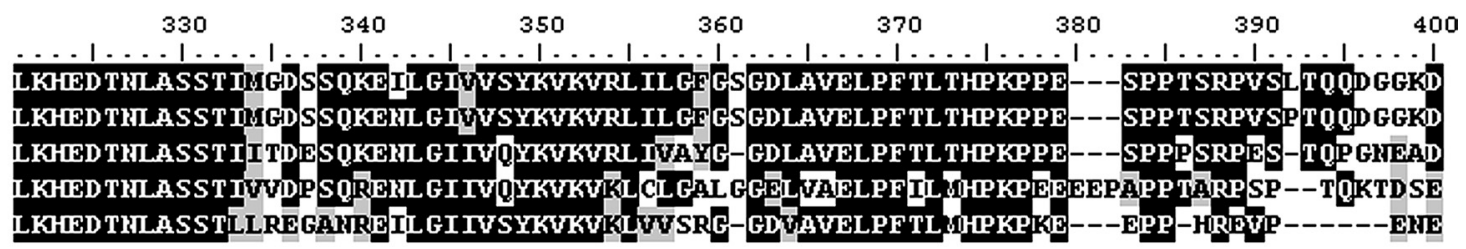

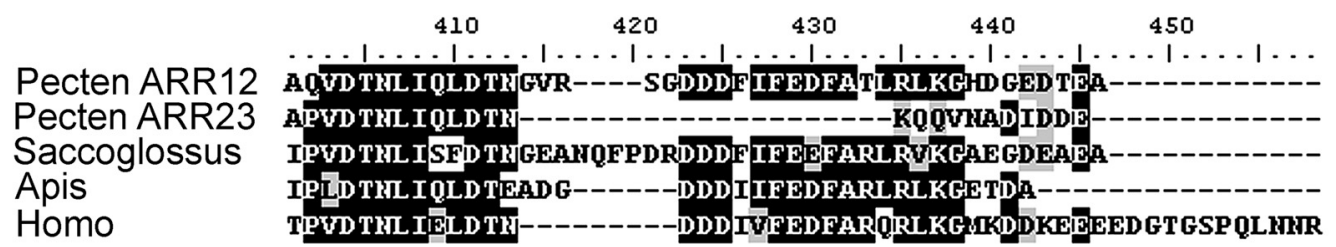

Figure 6. Amino acid sequence of two arrestin isoforms cloned from Pecten retina CDNA. A Clustal-W multialignment compares Arr12 and Arr23 (GenBank accession numbers HQ695998 and HQ695998, respectively) to the $\beta$-arrestin of Saccoglossus kowalevski (GenBank accession number XP_002736887.1), kurtz of Apis mellifera (GenBank accession number XP_392535.2), and $\beta$-arrestin 1B of Homo sapiens (GenBank accession NP_064647.1). Black shading indicates residue identity; similarity is indicated by gray shading.

basis of the only molluscan arrestin sequence reported (the squid, Loligo pealei) (Mayeenuddin and Mitchell, 2003), selecting amino acid blocks that appeared to be significantly conserved across other species according to a multisequence alignment. PCRs with two of the primer combinations tested yielded positive products, both $\approx 600 \mathrm{bp}$. After gel band excision, purification, cloning into pGEM and sequencing, a Blast-X search of the obtained nucleotide sequences identified both of them as arrestin $(E$ values of $1 e^{-85}$ to $\left.4 e^{-62}\right)$. Although the two sequences were mostly identical, they differed markedly in a single stretch of 71 contiguous nucleotides, as subsequently confirmed in 8 independent clones. Nondegenerate primers based on this diverging portion were then used in nested RACE reactions to retrieve the missing ends. The obtained extension products contained the two original amplicons, and displayed additional regions of divergence in both the $5^{\prime}$ and $3^{\prime}$ portions. By designing yet another primer set that exploited these differences, separate single-run amplifications confirmed that contiguous cDNAs were implicated in each case. The two full-length clones are hereafter referred to as Arr12 and Arr23, respectively. Arr12 is comprised of an ORF of $1275 \mathrm{nt}$; when translated, the 425 aa protein has a predicted molecular mass of $47 \mathrm{kDa}$ and $\mathrm{p} I$ calculated at 6.77. Arr23 is composed of an ORF of $1248 \mathrm{nt}$, which translates into a 416 aa protein with a predicted mass of $46 \mathrm{kDa}$ and theoretical $\mathrm{p} I$ of 5.94. The two translated sequences include the canonical $\mathrm{N}$ and $\mathrm{C}$-arrestin domains (N-domain AA 38-194; C-domain AA 213-372; Pfam); the overall identity is $81 \%$. For both isoforms the highest similarity, according to a Blast-p, is with the predicted 

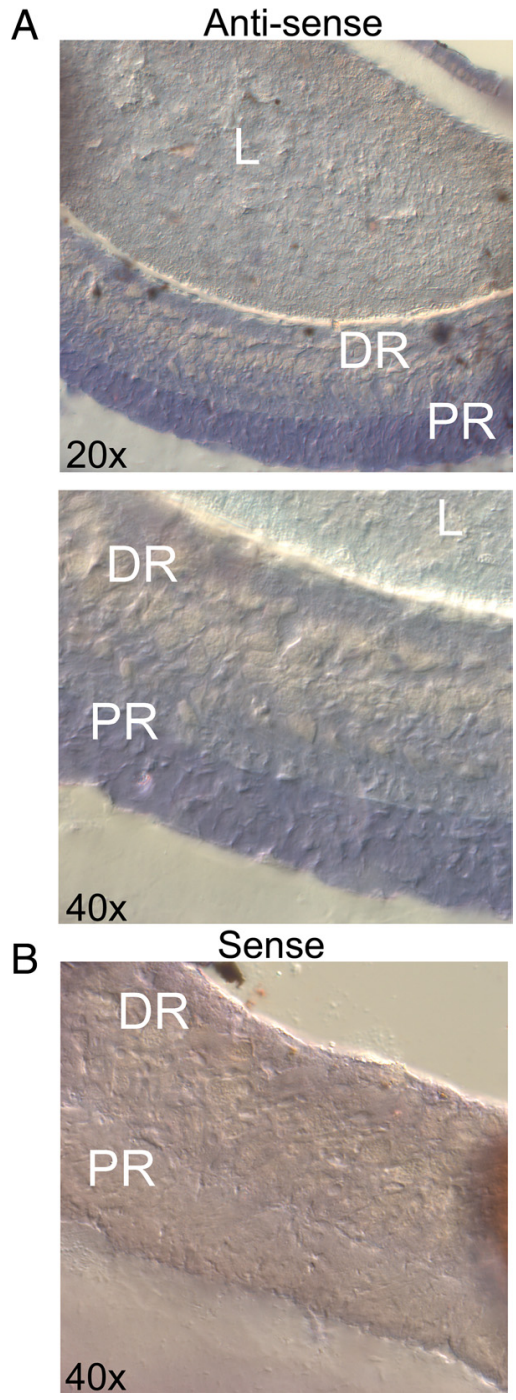

Figure 7. Localization of the arrestin transcripts in the retina of Pecten by in situ hybridization. $\boldsymbol{A}$, The micrographs illustrate a portion of a whole-eye cryosection incubated with Arr12 antisense probe, viewed at two magnifications. $\boldsymbol{B}$, Control section treated with the sense riboprobe. Only the antisense probe stained the tissue, the label being confined to the retina. However, both the ciliary and the rhabdomeric layers were decorated. L, Lens; DR, distal retina; $\mathrm{PR}$, proximal retina.

$\beta$-arrestin of the acorn worm, Saccoglossus kowalevskii (73\% and $67 \%$ identity, $E$ values $2 e^{-178}$ to $\left.2 e^{-157}\right)$; the similarity is quite high also with respect to human $\beta$-arrestin (63\% and $57 \%$ identity, $E$ values $2 e^{-150}$ to $\left.2 e^{-135}\right)$. In Figure 6 , a Clustal-W alignment shows the similarity to other reference arrestins.

The observations strongly support the coexistence of two distinct isoforms of arrestin in the retina of Pecten. We turned to in situ hybridization to localize the expression of these two arrestins. Purified arrestin amplicons that encompassed the divergent region originally identified were digested with endonucleases (HindIII and SpeI for Arr12, HindIII and SacII for Arr23); the size of the resulting restriction fragments was 665 and $441 \mathrm{bp}$, respectively. After gel purification, they were directionally ligated into the dual-promoter expression vector; digoxigenin-labeled RNA probes were then used on fixed whole-eye cryosections. Figure 7 reveals specific labeling by the Arr12 antisense probe, which clearly stained the retina of Pecten; however, the staining includes both the rhabdomeric and the ciliary photoreceptor layers. An
Arr23 riboprobe also decorated both the proximal and the distal retina (data not shown).

\section{Discussion}

Arrestins perform a critical function in the deactivation of various seven transmembrane receptors; the canonical scheme involves an initial phosphorylation of the receptor molecule, resulting in an increased affinity for binding arrestin, which in turn sterically hampers further interactions with G-proteins, thus preventing activation of their downstream effectors (for review, see Palczewski, 1994). In rods, it had long been known that rhodopsin is phosphorylated in a light-dependent manner (Bownds et al., 1972; Kühn and Dreyer, 1972), and that only the phosphorylated form of the photopigment can subsequently bind to arrestin (Kühn et al., 1984). The molecular identity of the kinase was subsequently established as a member of the G-coupled receptor kinase family (GRK), which mediates homologous desensitization by targeting stimulus-activated receptors (Lorenz et al., 1991)

In microvillar photoreceptors rhodopsin also becomes phosphorylated after illumination (Paulsen and Hoppe, 1978; Vandenberg and Montal, 1984; Bentrop and Paulsen, 1986; Byk et al., 1993), and a rhodopsin kinase has been isolated from octopus (Kikkawa et al., 1998) squid (Mayeenuddin and Mitchell, 2001) and Drosophila (Doza et al., 1992; Lee et al., 2004). However, the role of rhodopsin phosphorylation in shaping the interaction with arrestin seemingly diverges from rods and cones. For example, in preparations of squid microvillar membrane, phosphorylation of metarhodopsin does not appear to be necessary for the binding of arrestin (Bentrop et al., 1993; Plangger et al., 1994), and in fact ATP is not absolutely required for termination of light-activated GTPase activity (Kahana et al., 1992). Additionally, unlike in vertebrates, arrestin itself undergoes rapid phosphorylation following light stimulation (Wiebe et al., 1989; Matsumoto et al., 1994; Mayeenuddin and Mitchel, 2003). In Drosophila and Limulus this effect is mediated by a Ca/ calmodulin-dependent protein kinase II (Edwards et al., 1989; Wiebe et al., 1989; Matsumoto et al., 1994; Calman et al., 1996; Kahn and Matsumoto, 1997). By contrast, in squid a rhodopsin kinase (dubbed SQRK) fulfills the role (Mayeenuddin and Mitchel, 2003). In all cases, however, the phosphorylation of arrestin is calcium dependent, a phenomenon that dovetails with the large increase in cytosolic calcium that accompanies the light response of microvillar photoreceptors. A simple scheme has been proposed, in which a photo-induced rise in Ca leads to phosphorylation of arrestin and downregulation of the cascade (Scott et al., 1997). However, other evidence suggests instead that phosphorylation of arrestin is critical for its release from rhodopsin (Alloway and Dolph, 1999).

Invertebrate visual arrestins have been molecularly identified only in a few species, like Limulus, Drosophila, Calliphora, and Loligo (Hyde et al., 1990; LeVine et al., 1990; Smith et al., 1990; Yamada et al., 1990; Plangger et al., 1994; Smith et al., 1995; Mayeenuddin and Mitchel, 2003). Moreover, only a few studies have examined their impact either on the biochemistry of the transduction cascade (Byk et al., 1993) or on the visual response in vivo (Dolph et al., 1993). In the present report we have shown that arrestin expresses in molluscan hyperpolarizing ciliary photoreceptors and is implicated in the termination of the photocurrent. These visual receptors comprise a third lineage of light-sensing cells-distinct from both the rods and cones of vertebrates and the microvillar receptors of invertebrates-and use a disparate transduction scheme, more reminiscent of che- 
moreceptors of the olfactory epithelium than other visual cells. In addition, functional impairment of the arrestin pool resulted in an enhanced propensity to trigger sustained excitation, even in the absence of large metarhodopsin accumulation, but could be reset by photoconversion $\mathrm{M} \rightarrow \mathrm{R}$; this observation strengthens the contention that prolonged aftercurrents evoked by intense chromatic illumination reflect saturation of arrestin population; in fact, reduction of the rhodopsin/arrestin molar ratio in Drosophila eliminates the phenomenon (Dolph et al., 1993).

Molecular cloning of Pecten retina arrestin reveals the presence of two distinct isoforms; both present the canonical organization consisting of two homologous domains ( $\mathrm{N}$-arrestin and C-arrestin), separated by a short intervening "hinge" region. Sequence comparison indicates a higher similarity to $\beta$-arrestin than to conventional visual arrestins of vertebrates, but lack the signature clathrin-binding LIAF motif in the $\mathrm{C}$ terminus (for review, see Gurevich and Gurevich, 2006); this is consistent with the formation of a transient complex with the photopigment without promoting its endocytosis, a notion that is in line with the dynamic regulation of the visual response, as it occurs in other photoreceptor types, instead of the mediation of receptor turnover. Arrestins isolated from the eye of squid, horseshoe crab, and fly also resemble $\beta$-arrestin more closely than vertebrate visual arrestin. In this respect, it is worth pointing out that the traditional segregation of visual and nonvisual arrestins has proved faulty: for example, in Anopheles gambia and Drosophila, Arr1 and Arr2, previously thought to be strictly visual arrestins, localize also in the chemosensory antennae (Merrill et al., 2002); moreover, their knock-out functionally impacts the olfactory response.

Each of the two light-transducing cell types of the eye of Pecten (rhabdomeric in the proximal retina and ciliary in the distal retina) requires some mechanism of photopigment inactivation, and one could have surmised that the two arrestin clones are associated with the two classes of photoreceptors. Although some antibodies selectively labeled the distal retina of Pecten, where ciliary photoreceptors are located (Fig. 2), such a segregation was not evident from in situ hybridization assays; therefore, the possibility of differential expression of Arr12 and Arr23 remains unclear at this point. One must reckon, however, that the identity of the two arrestin isoforms over much of their nucleotide sequence inevitably limits the relative differences that can exist between probes of adequate size (the ones tested diverged in only 57 bases, which amounts to only $\approx 9-13 \%$ ), and this is likely to hinder differential hybridization. Alternative approaches that could help settle this issue in a more definitive manner in the future entail raising de novo antibodies against the divergent stretches of the two clones (assuming that these are immunogenic); the antibodies used in the present study do not provide such detailed information because for those raised against defined peptides the sequence has not been disclosed by the manufacturer. Perhaps, single-cell PCR could also provide a suitable avenue.

The detailed mechanisms by which arrestin regulates the visual response-and in turn is regulated-in ciliary invertebrate visual cells remain to be elucidated. Very limited information is available on the photopigment of this class of visual cells, which has only been molecularly identified in one species (Patinopecten yessoensis) (Kojima et al., 1997). At this point it is not known whether it is phosphorylated in a light-dependent manner, and, if so, the identity of the kinase implicated. Likewise, considering that this unusual cascade partakes of both vertebrate and invertebrate light-signaling schemes, it has yet to be determined whether or not pigment phosphorylation is required for interaction with arrestin-an aspect where photoreceptors of the two phyla depart. As for the regulation of arrestin itself by phosphorylation, one would anticipate a strong divergence with respect to microvillar photoreceptors, where in all documented cases this process is strongly calcium dependent; by contrast, in ciliary cells cytosolic calcium does not change measurably during the photoresponse (Gomez and Nasi, 2005), as they lack the lightdependent machinery for both Ca mobilization from internal stores (Gomez and Nasi, 1995) and influx through the plasma membrane (Nasi and Gomez, 1999).

The striking variety in morphological design of light-sensing organs across animals had inspired the traditional view that the machinery subserving visual functions in different phyla-including the two canonical lineages of photoreceptor cells-must have evolved independently (Salvini-Plawen and Mayr, 1977). More recent molecular studies have challenged such position, by revealing universal master control genes for eye development (Gehring, 2002) and the recurrence of a stereotyped general scheme for light transduction (Koyanagi et al., 2008). Specific classes of signaling elements are ubiquitous both in rods and cones as well as in microvillar photoreceptors: (1) a photopigment endowed with a retinal-based chromophore, (2) a G-protein conveying the light signal, and (3) an arrestin to terminate their interaction. The present results show that in ciliary invertebrate photoreceptors, which comprise a recently uncovered lineage of visual cells, light response inactivation also entails the recruitment of an arrestin to decouple rhodopsin from its cognate G-protein. A recent bioinformatics search across lower phyla identified $\beta$ /visual-arrestin in cnidaria (Alvarez, 2008); the earliest cilia-bearing photoreceptors can be traced to cubozoa jellyfish, which belong to this phylum (Martin, 2002), so that the origin of such lineage of photoreceptors predates bilateria. The ancient origin and similarity of the shutoff mechanisms that operate across all classes of light-sensing cells described so far in animals fits well with the notion of a monophyletic origin of visual mechanisms.

\section{References}

Alloway PG, Dolph PJ (1999) A role for the light-dependent phosphorylation of visual arrestin. Proc Natl Acad Sci U S A 96:6072-6077.

Alvarez CE (2008) On the origins of arrestin and rhodopsin. BMC Evol Biol 8:222.

Barber VC, Evans EM, Land MF (1967) The fine structure of the eye of the mollusc Pecten maximus. Z Zellforsch Mikrosk Anat 76:295-312.

Bentrop J, Paulsen R (1986) Light-modulated ADP-ribosylation, protein phosphorylation and protein binding in isolated fly photoreceptor membranes. Eur J Biochem 161:61-67.

Bentrop J, Plangger A, Paulsen R (1993) An arrestin homolog of blowfly photoreceptors stimulates visual-pigment phosphorylation by activating a membrane-associated protein kinase. Eur J Biochem 216:67-73.

Bownds D, Dawes J, Miller J, Stahlman M (1972) Phosphorylation of frog photoreceptor membranes induced by light. Nat New Biol 237:125-127.

Byk T, Bar-Yaacov M, Doza YN, Minke B, Selinger Z (1993) Regulatory arrestin cycle secures the fidelity and maintenance of the fly photoreceptor cell. Proc Natl Acad Sci U S A 90:1907-1911.

Calman BG, Andrews AW, Rissler HM, Edwards SC, Battelle BA (1996) Calcium/calmodulin-dependent protein kinase II and arrestin phosphorylation in Limulus eyes. J Photochem Photobiol B 35:33-44.

Cornwall MC, Gorman AL (1983) Colour dependence of the early receptor potential and late receptor potential in scallop distal photoreceptor. J Physiol 340:307-334.

Dolph PJ, Ranganathan R, Colley NJ, Hardy RW, Socolich M, Zuker CS (1993) Arrestin function in inactivation of G protein-coupled receptor rhodopsin in vivo. Science 260:1910-1916.

Doza YN, Minke B, Chorev M, Selinger Z (1992) Characterization of fly rhodopsin kinase. Eur J Biochem 209:1035-1040.

Edwards SC, Wishart AC, Wiebe EM, Battelle BA (1989) Light-regulated proteins in Limulus ventral photoreceptor cells. Vis Neurosci 3:95-105.

Fain GL, Hardie R, Laughlin SB (2010) Phototransduction and the evolution of photoreceptors. Curr Biol 20:R114-R124. 
Gehring WJ (2002) The genetic control of eye development and its implications for the evolution of the various eye-types. Int J Dev Biol 46:65-73.

Gomez M, Nasi E (1995) Activation of light-dependent potassium channels in ciliary invertebrate photoreceptors involves cGMP but not the IP3/Ca 2 cascade. Neuron 15:607-618.

Gomez M, Nasi E (2005) Calcium-independent, cGMP-mediated light adaptation in ciliary photoreceptors. J Neurosci 25:2042-2049.

Gomez MP, Nasi E (1994) The light-sensitive conductance of hyperpolarizing invertebrate photoreceptors: a patch-clamp study. J Gen Physiol 103:939-956.

Gomez MP, Nasi E (2000) Light transduction in invertebrate hyperpolarizing photoreceptors: possible involvement of a $\mathrm{G}_{\mathrm{o}}$-regulated guanylate cyclase. J Neurosci 20:5254-5263.

Gorman AL, McReynolds JS (1969) Hyperpolarizing and depolarizing receptor potentials in the scallop eye. Science 165:309-310.

Gorman AL, McReynolds JS (1978) Ionic effects on the membrane potential of the hyperpolarizing photoreceptors in scallop retina. J Physiol 275: 345-355.

Gurevich EV, Gurevich VV (2006) Arrestins: ubiquitous regulators of cellular signaling pathways. Genome Biol 7:236.1-236.8.

Hardie RC (2003) Phototransduction: shedding light on translocation. Curr Biol 13:R775-R777.

Hillman P, Hochstein S, Minke B (1983) Transduction in invertebrate photoreceptors: role of pigment bistability. Physiol Rev 63:668-772.

Hyde DR, Mecklenburg KL, Pollock JA, Vihtelic TS, Benzer S (1990) Twenty Drosophila visual system cDNA clones, one is a homolog of human arrestin. Proc Natl Acad Sci U S A 87:1008-1012.

Kahana A, Robinson PR, Lewis LJ, Szuts EZ, Lisman JE (1992) ATPindependent deactivation of squid rhodopsin. Vis Neurosci 9:595-602.

Kahn ES, Matsumoto H (1997) Calcium/calmodulin-dependent kinase II phosphorylates Drosophila visual arrestin. J Neurochem 68:169-175.

Kikkawa S, Yoshida N, Nakagawa M, Iwasa T, Tsuda M (1998) A novel rhodopsin kinase in octopus photoreceptor possesses a pleckstrin homology domain and is activated by G protein $\beta \gamma$-subunits. J Biol Chem 273:7441-7447.

Kojima D, Terakita A, Ishikawa T, Tsukahara Y, Maeda A, Shichida Y (1997) A novel $\mathrm{G}_{\mathrm{o}}$-mediated phototransduction cascade in scallop visual cells. J Biol Chem 272:22979-22982.

Koyanagi M, Takano K, Tsukamoto H, Ohtsu K, Tokunaga F, Terakita A (2008) Jellyfish vision starts with cAMP signaling mediated by opsin- $\mathrm{G}_{\mathrm{s}}$ cascade. Proc Natl Acad Sci U S A 105:15576-15580.

Kühn H, Dreyer WJ (1972) Light dependent phosphorylation of rhodopsin by ATP. FEBS Lett 20:1-6.

Kühn H, Hall SW, Wilden U (1984) Light-induced binding of 48 kDaprotein to photoreceptor membranes is enhanced by phosphorylation of rhodopsin. FEBS Lett 176:473-478.

Lee SJ, Xu H, Montell C (2004) Rhodopsin kinase activity modulates the amplitude of the visual response in Drosophila. Proc Natl Acad Sci U S A 101:11874-11879.

LeVine H 3rd, Smith DP, Whitney M, Malicki DM, Dolph PJ, Smith GF, Burkhart W, Zuker CS (1990) Isolation of a novel visual-system-specific arrestin, an in vivo substrate for light-dependent phosphorylation. Mech Dev 33:19-25.

Lorenz W, Inglese J, Palczewski K, Onorato JJ, Caron MG, Lefkowitz RJ
(1991) The receptor kinase family: primary structure of rhodopsin kinase reveals similarities to the beta-adrenergic receptor kinase. Proc Natl Acad Sci U S A 88:8715-8719.

Martin VJ (2002) Photoreceptors of cnidarians. Can J Zool 80:1703-1722.

Matsumoto H, Kurien BT, Takagi Y, Kahn ES, Kinumi T, Komori N, Yamada T, Hayashi F, Isono K, Pak WL, Jackson KW, Tobin SL (1994) Phosrestin I undergoes the earliest light-induced phosphorylation by a calcium/ calmodulin-dependent protein kinase in Drosophila photoreceptors. Neuron 12:997-1010.

Mayeenuddin LH, Mitchell J (2001) cDNA cloning and characterization of a novel squid rhodopsin kinase encoding multiple modular domains. Vis Neurosci 18:907-915.

Mayeenuddin LH, Mitchell J (2003) Squid visual arrestin: cDNA cloning and calcium-dependent phosphorylation by rhodopsin kinase (SQRK). J Neurochem 85:592-600.

Merrill CE, Riesgo-Escovar J, Pitts RJ, Kafatos FC, Carlson JR, Zwiebel LJ (2002) Visual arrestins in olfactory pathways of Drosophila and the malaria vector mosquito Anopheles gambiae. Proc Natl Acad Sci U S A 99:1633-1638.

Miller WH (1958) Derivatives of cilia in the distal sense cells of the retina of Pecten. J Biophys Biochem Cytol 4:227-228.

Nasi E, Gomez M (1999) Divalent cation interactions with light-dependent K channels: kinetics of voltage-dependent block and requirement for an open pore. J Gen Physiol 114:653-672.

Palczewski K (1994) Structure and functions of arrestins. Protein Sci 3:1355-1361.

Paulsen R, Hoppe I (1978) Light-activated phosphorylation of cephalopod rhodopsin. FEBS Lett 96:55-58.

Plangger A, Malicki D, Whitney M, Paulsen R (1994) Mechanism of arrestin 2 function in rhabdomeric photoreceptors. J Biol Chem 269:26969-26975.

Salvini-Plawen LV, Mayr E (1977) On the evolution of photoreceptors and eyes. In: Evolutionary biology, Vol 10 (Hecht MK, Steere WC, Wallace B eds), pp 207-263. New York: Plenum.

Scott K, Sun Y, Beckingham K, Zuker CS (1997) Calmodulin regulation of Drosophila light-activated channels and receptor function mediates termination of the light response in vivo. Cell 91:375-383.

Smith DP, Shieh BH, Zuker CS (1990) Isolation and structure of an arrestin gene from Drosophila. Proc Natl Acad Sci U S A 87:1003-1007.

Smith WC, Greenberg RM, Calman BG, Hendrix MM, Hutchinson L, Donoso LA, Battelle BA (1995) Isolation and expression of an arrestin cDNA from the horseshoe crab lateral eye. J Neurochem 64:1-13.

Vandenberg CA, Montal M (1984) Light-regulated biochemical events in invertebrate photoreceptors. 2. Light-regulated phosphorylation of rhodopsin and phosphoinositides in squid photoreceptor membranes. Biochemistry 23:2347-2352.

Wiebe EM, Wishart AC, Edwards SC, Battelle BA (1989) Calcium/ calmodulin-stimulated phosphorylation of photoreceptor proteins in Limulus. Vis Neurosci 3:107-118.

Yamada T, Takeuchi Y, Komori N, Kobayashi H, Sakai Y, Hotta Y, Matsumoto H (1990) 49-kilodalton phosphoprotein in the Drosophila photoreceptor is an arrestin homolog. Science 248:483-486.

Yau KW, Hardie RC (2009) Phototransduction motifs and variations. Cell 139:246-264. 